\title{
A FINITE ELEMENT ANALYSIS OF AXISYMMETRIC FLOW OF AIR THROUGH THE TOP RISER OF CASTING USING THE STREAM FUNCTION MODEL
}

\author{
F. Inegbedion ${ }^{1,}{ }^{*}$ and J. A. Akpobi ${ }^{2}$

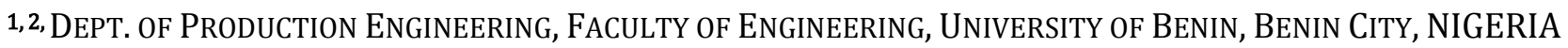 \\ E-mail addresses: 1 francis.inegbedion@uniben.edu, ${ }^{2}$ john.akpobi@uniben.edu
}

\begin{abstract}
Casting is a manufacturing process for making complex shapes of metal materials. Casting has two stages - filling process requiring a gating system and solidification process requiring a riser. The air in the mould cavity during casting is displaced through the riser by the molten metal in a very rapid manner necessitating the need to understand the air flow behaviour as it exit through the riser. The finite element method and the stream function model were used to analyze the axisymmetric flow of air through the top risers of casting. Results show that the velocity profile at any cross section is parabolic in shape with the maximum velocity at the centre. Comparing results with exact solution shows that the finite element result converged towards the exact solution.
\end{abstract}

Keywords: stream function, axisymmetric flow, top riser, air, casting

\section{NOMENCLATURE}

$\begin{array}{ll}\mathrm{D} & \text {-Riser diameter } \\ f^{e} & \text {-Nodal forces } \\ \mathrm{H} & \text {-Riser height } \\ K^{e} & \text {-Stiffness matrix } \\ \mathrm{N} & \text {-Interpolation function } \\ r \& Z & \text {-Axis-symmetry coordinates variables } \\ \mathrm{U}_{0} & \text {-Initial Velocity of Air } \\ \mathrm{U}_{1} & \text {-Final Velocity of Air } \\ u, V, W & \text {-r } \theta, \quad Z \text { components of the velocity } \\ & \text { respectively } \\ u_{r} \& u_{z} & \text {-Axis-symmetry velocity components } \\ \rho & \text {-Density } \\ \psi & \text {-Stream function }\end{array}$

\section{INTRODUCTION}

A key element in producing quality castings is the proper design and sizing of the gating and riser systems. A foundry can produce the best quality moulds, cores and molten metal and still end up with a poor quality casting by using poorly designed gating and riser systems. The main objective of a gating system is to lead clean molten metal poured from ladle to the casting cavity, ensuring smooth, uniform and complete filling. [1]. A riser is a reservoir built into a metal casting mould to prevent cavities due to shrinkage, because metals are less dense as liquids than as solids (with some exceptions). This can leave a void, generally at the last point to solidify. Risers prevent this by providing molten metal at the point of likely shrinkage, so that the cavity forms in the riser, not the casting [2]. The riser apart from serving as a reservoir to compensate for shrinkage during solidification, also serve as a channel through which the air displaced as a result of filling the mould cavity with the molten metal goes out of the mould cavity [3]. Numerous efforts have been made by castings engineers and researchers on gating system design over the past few decades [4 - 9]. Although there are general casting design rules and empirical equations for the gating ratio, pouring time, gating and riser system dimensions and optimization [5, 7], consideration has not been given prior to now to the air flow behaviour in the casting mould. Hence this work focuses on the analysis of the air flow through the top risers of casting using the stream function model.

\section{FINITE ELEMENT ANALYSIS}

\subsection{The Model Equation}

The model equation is the stream function model of axisymmetric flow of air in casting mould [13]

$$
\frac{\partial^{2} \psi}{\partial z^{2}}=-\frac{\partial^{2} \psi}{\partial r^{2}}
$$

2.2Weak Formulation of the Model Equation

The weighted residual of equation (1) is 


$$
N_{i}(r, z)\left(\frac{\partial^{2} \psi}{\partial z^{2}}-\frac{\partial^{2} \psi}{\partial r^{2}}\right)=0
$$

We integrated equation (2) over the element domain $\Omega_{e}$

$$
\begin{gathered}
\int_{\Omega_{e}} N_{i}(r, z)\left(\frac{\partial^{2} \psi}{\partial z^{2}}+\frac{\partial^{2} \psi}{\partial r^{2}}\right) d r d z=\int_{\Omega_{e}} N\left(\frac{\partial^{2} \psi}{\partial z^{2}}\right) d r d z+ \\
\int_{\Omega_{e}} N\left(\frac{\partial^{2} \psi}{\partial r^{2}}\right) d r d z=\int_{\Omega_{e}} N\left(\frac{\partial}{\partial z}\right)\left(\frac{\partial \psi}{\partial z}\right) d r d z+ \\
\int_{\Omega_{e}} N\left(\frac{\partial}{\partial r}\right)\left(\frac{\partial \psi}{\partial r}\right) d r d z=0 \\
\operatorname{let} \frac{\partial \psi}{\partial r}=\frac{\partial \psi}{\partial z}=H \\
\therefore \int_{\Omega_{e}} N\left(\frac{\partial H}{\partial z}\right) d r d z+\int_{\Omega_{e}} N\left(\frac{\partial H}{\partial r}\right) d r d z=0
\end{gathered}
$$

We integrated equation (5) by parts with respect to $Z$ and $r$ using the basic relation

$$
\begin{aligned}
\int_{\Omega_{e}} u d v & =-\int_{\Omega_{e}} u d v+\left.u v\right|_{\Omega_{e}} \\
\int_{\Omega_{e}} N\left(\frac{\partial^{2} \psi}{\partial z^{2}}\right) d r d z & \\
= & -\int_{\Omega_{e}} N\left(\frac{\partial \psi}{\partial z}\right)\left(\frac{\partial N}{\partial z}\right) d r d z \\
& +\int_{T_{2}}^{T_{2}} N \frac{\partial \psi}{\partial r} n_{r} d \Omega_{e} \\
\int_{\Omega_{e}} N\left(\frac{\partial^{2} \psi}{\partial z^{2}}\right) d r d z & \\
& =-\int_{\Omega_{e}}\left(\frac{\partial \psi}{\partial r}\right)\left(\frac{\partial N}{\partial r}\right) d r d z \\
& +\int_{T_{2}}^{T_{2}} N \frac{\partial \psi}{\partial r} n_{r} d \Omega_{e}
\end{aligned}
$$

We Combined equations (7) and (8) using equation (3) to give the integration by parts.

$$
\begin{gathered}
\int_{\Omega_{e}}\left(\left(\frac{\partial[N]}{d z}\right)\left(\frac{\partial N}{\partial z}\right)+\left(\frac{\partial[N]}{d r}\right)\left(\frac{\partial N}{\partial r}\right)\right) d r d z\{\Psi\} \\
=\int_{T_{1}}^{T_{2}} N\left(u n_{z}-w n_{r}\right) d \Omega_{e}
\end{gathered}
$$

\subsection{The Finite Element Model}

The finite element model of equation (9) is given in matrix form as equation (10):

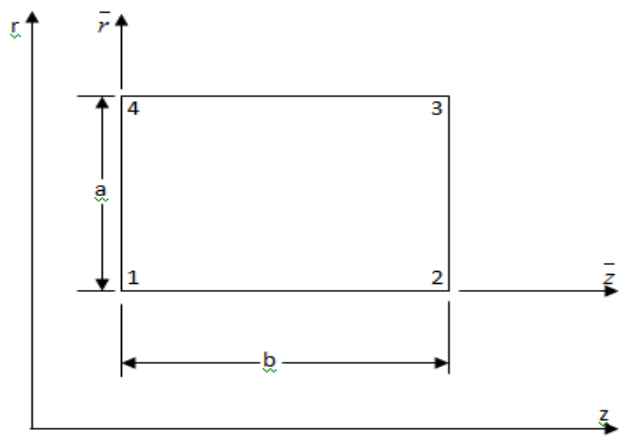

Figure 1a: Geometry of the element

$$
\begin{gathered}
{\left[k^{e}\right]\{\psi\}=\left|f^{e}\right|} \\
{\left[k^{e}\right]=\int_{\Omega_{e}}\left(\left(\frac{\partial[N]}{d z}\right)\left(\frac{\partial N}{\partial z}\right)\right.} \\
\left.+\left(\frac{\partial[N]}{d r}\right)\left(\frac{\partial N}{\partial r}\right)\right) d r d z \\
\left|\int^{e}\right|=\int_{T_{1}}^{T_{2}} N\left(u n_{z}-w n_{r}\right) d \Omega\left|f^{e}\right|
\end{gathered}
$$

Let $\left(u n_{z}-w n_{r}\right)=U_{1}$. Therefore,

$$
\left|f^{e}\right|=\int_{T_{1}}^{T_{2}} N\left(U_{1}\right) d \Omega
$$

\subsection{Derivation of the Finite Element Interpolation Functions}

The stream function model over the domain of interest is discretized into finite elements having $M$ nodes, using suitable interpolation model for $\psi^{(e)}$ in element $e$ as [11]:

$$
\psi \approx \psi(r, z)=\sum_{i=1}^{M} N_{i}(r, z) \psi_{i}=[N]\{\psi\}
$$

The interpolation functions $N_{i}(r, z)$ are the same as those developed for linear rectangular elements, with $x$ $=r$ and $y=z$ [13]. This will enable us to evaluate the integrals of the $K_{i j}^{e}$ and $f_{i j}^{e}$. Let's consider an approximation of the form:

$$
N(r, z)=c_{1}+c_{2} r+C_{3}+C_{4} r z
$$

and use a rectangular element with sides $a$ and $b$ (Figure 1a).

We choose a local coordinate system $(a, b)$ to derive the interpolation function. Thus equation (15) becomes

$$
N(r, z)=c_{1}+c_{2} a+c_{3} b+c_{4} a b
$$

and require

$$
\begin{aligned}
N_{1}=N(0,0)=c_{1} & ; N_{2}=N(a, 0)=c_{1}+c_{2} a N_{3} \\
= & N(a, b)=c_{1}+c_{2} a+c_{3} b+c_{4} a b N_{4} \\
= & N(0, b)=c_{1}+c_{3} b
\end{aligned}
$$

Solving for $c_{i}(i=1, \ldots, 4)$, in equations (17) we obtained the following

$$
\begin{gathered}
c_{1}=N_{1} ; \quad c_{2}=\frac{N_{2}-N_{1}}{a} ; \quad c_{3}=\frac{N_{4}-N_{1}}{b} \\
c_{4}=\frac{N_{3}-N_{4}+N_{1}-N_{1}}{a b}
\end{gathered}
$$

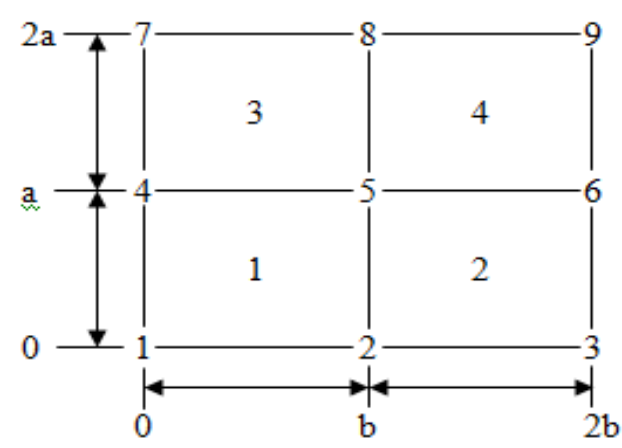

Figure 1b: Four Linear Rectangular Elements 
We substituted equation (18) into equation (16) and noting that $a=r$ and $b=z$, we obtained

$$
\begin{array}{r}
N(r, z)=\left(1-\frac{r}{a}\right)\left(1-\frac{z}{b}\right) N_{1}+\left(1-\frac{z}{b}\right) N_{2}+\frac{r z}{a b} N_{3}+\frac{z}{b}\left(1-\frac{r}{a}\right) N_{4}=\phi_{1} N_{1}+\phi_{2} N_{2}+\phi_{3} N_{3}+\phi_{4} N_{4} \\
\phi_{1}\left(1-\frac{r}{a}\right)\left(1-\frac{z}{b}\right) \quad \phi_{2}=\frac{r}{a}\left(1-\frac{z}{b}\right) \quad \phi_{3}=\frac{r z}{a b} \quad \phi_{4}=\frac{z}{b}\left(1-\frac{r}{a}\right)
\end{array}
$$

We differentiated equations (20) with respect to $r$ and $z$

$$
\begin{aligned}
\frac{d \phi_{1}}{d r}=\left(-\frac{1}{a}\right)\left(1-\frac{z}{b}\right) & =\left(\frac{-1}{a}+\frac{z}{a b}\right) ; \frac{d \phi_{1}}{d z}=\left(-\frac{1}{b}\right)\left(1-\frac{r}{a}\right)=\left(\frac{-1}{b}+\frac{r}{a b}\right) ; \frac{d \phi_{2}}{d r}=\frac{1}{a}\left(1-\frac{z}{b}\right)=\frac{1}{a}-\frac{z}{a b} \\
\frac{d \phi_{2}}{d z} & =\frac{r}{a b} ; \frac{d \phi_{3}}{d r}=\frac{z}{a b} ; \frac{d \phi_{3}}{d z}=\frac{r}{a b} ; \quad \frac{d \phi_{4}}{d r}=-\frac{z}{a b} ; \quad \frac{d \phi_{4}}{d z}=\frac{1}{b}\left(1-\frac{r}{a}\right)=-\frac{r}{a b}+\frac{1}{b}
\end{aligned}
$$

\subsection{Evaluation of Finite Elements to obtain the Global System of Algebraic Equations}

We rewrote $\left[\mathrm{Ke}^{\mathrm{e}}\right]$ in equation (11) as the sum of four basic matrices and using the interpolation function of (21) evaluated the several $K_{i j}^{e}$ of each matrix using Figures $1 \mathrm{a}$ and $1 \mathrm{~b}$

$$
\begin{gathered}
K_{i j}^{e}=\left(K^{1}+K^{2}+K^{3}+K^{4}\right) \\
K^{1}=\left[\begin{array}{cccc}
K_{11}^{1} & K_{12}^{1} & K_{13}^{1} & K_{14}^{1} \\
K_{21}^{1} & K_{22}^{1} & K_{23}^{1} & K_{24}^{1} \\
K_{31}^{1} & K_{32}^{1} & K_{33}^{1} & K_{34}^{1} \\
K_{41}^{1} & K_{42}^{1} & K_{43}^{1} & K_{44}^{1}
\end{array}\right] \quad K^{2}=\left[\begin{array}{cccc}
K_{11}^{2} & K_{12}^{2} & K_{13}^{2} & K_{14}^{2} \\
K_{21}^{2} & K_{22}^{2} & K_{23}^{2} & K_{24}^{2} \\
K_{31}^{2} & K_{32}^{2} & K_{33}^{2} & K_{34}^{2} \\
K_{41}^{2} & K_{42}^{2} & K_{43}^{2} & K_{44}^{2}
\end{array}\right] \text { similarly for } K^{3} \text { and }^{4} \\
K_{11}^{1}=\int_{0}^{b} \int_{0}^{a}\left[\left(\left(-\frac{1}{a}\right)\left(-\frac{z}{b}\right)\right)^{2}+\left(\left(-\frac{1}{b}\right)\left(-\frac{r}{a}\right)\right)^{2}\right] d r d z=\frac{a}{3 b}+\frac{b}{3 a} \\
K_{12}^{1}=\int_{0}^{b} \int_{0}^{a}\left[\left(-\frac{1}{a}+\frac{z}{a b}\right)^{2}+\left(-\frac{1}{a}+\frac{r}{a b}\right)\left(-\frac{r}{a b}\right)\right] d r d z=\frac{a}{6 b}+\frac{b}{3 a}
\end{gathered}
$$

Similarly we evaluated all elements of $K^{1}$ matrix

$$
\begin{aligned}
& K_{11}^{2}=\int_{0}^{b} \int_{a}^{2 a}\left[\left(\left(-\frac{1}{a}\right)\left(-\frac{z}{b}\right)\right)^{2}+\left(\left(-\frac{1}{b}\right)\left(-\frac{r}{a}\right)\right)^{2}\right] d r d z=\frac{a}{3 b}+\frac{b}{3 a} \\
& K_{12}^{2}=\int_{0}^{b} \int_{a}^{2 a}\left[\left(-\frac{1}{a}+\frac{z}{a b}\right)^{2}+\left(-\frac{1}{a}+\frac{r}{a b}\right)\left(-\frac{r}{a b}\right)\right] d r d z=\frac{b}{3 a}+\frac{5 a}{6 b}
\end{aligned}
$$

Similarly we evaluated all elements of $K^{2}$ matrix

$$
\begin{gathered}
K_{11}^{3}=\int_{0}^{2 b} \int_{a}^{a}\left[\left(\left(-\frac{1}{a}\right)\left(1-\frac{z}{b}\right)\right)^{2}+\left(\left(-\frac{1}{b}\right)\left(1-\frac{r}{a}\right)\right)^{2}\right] d r d z=\frac{a}{3 b}+\frac{b}{3 a} \\
K_{12}^{3}=\int_{b}^{2 b} \int_{0}^{a}\left[\left(-\frac{1}{a}+\frac{z}{a b}\right)^{2}+\left(-\frac{1}{b}+\frac{r}{a b}\right)\left(-\frac{r}{a b}\right)\right] d r d z=\frac{a}{6 b}+\frac{b}{3 a}
\end{gathered}
$$

Similarly we evaluated all elements of $K^{3}$ matrix

$$
\begin{gathered}
K_{11}^{4}=\int_{b}^{2 b} \int_{a}^{2 a}\left[\left(\left(-\frac{1}{a}\right)\left(1-\frac{z}{b}\right)\right)^{2}+\left(\left(-\frac{1}{b}\right)\left(1-\frac{r}{a}\right)\right)^{2}\right] d r d z=\frac{a}{3 b}+\frac{b}{3 a} \\
K_{12}^{4}=\int_{b}^{2 b} \int_{a}^{2 a}\left[\left(-\frac{1}{a}+\frac{z}{a b}\right)^{2}+\left(-\frac{1}{b}+\frac{r}{a b}\right)\left(-\frac{r}{a b}\right)\right] d r d z=\frac{-5 a}{6 b}+\frac{b}{3 a}
\end{gathered}
$$

Similarly we evaluated all elements of $K^{4}$ matrix

Equation (22) became 


$$
\left[K^{e}\right]=\left[\begin{array}{c}
{\left[\begin{array}{ccccc}
\frac{a}{3 b}+\frac{b}{3 a} & \frac{a}{6 b}+\frac{b}{3 a} & \frac{-a}{6 b}-\frac{b}{6 a} & \frac{a}{3 b}+\frac{b}{6 a} \\
\frac{a}{6 b}+\frac{b}{6 a} & \frac{a}{3 b}+\frac{b}{3 a} & \frac{b}{6 a}-\frac{a}{3 b} & \frac{b}{6 a}-\frac{a}{6 b} \\
\frac{-a}{6 b}-\frac{b}{6 a} & \frac{b}{6 a}-\frac{a}{3 b} & \frac{a}{3 b}+\frac{b}{3 a} & \frac{a}{6 b}-\frac{a}{3 a} \\
\frac{a}{3 b}+\frac{b}{6 a} & \frac{b}{6 a}-\frac{a}{6 b} & \frac{a}{6 b}-\frac{b}{3 a} & \frac{a}{3 b}+\frac{b}{3 a}
\end{array}\right]+\left[\begin{array}{cccc}
\frac{a}{3 b}+\frac{b}{3 a} & \frac{b}{3 a}-\frac{5 a}{6 b} & \frac{5 a}{6 b}-\frac{b}{6 a} & \frac{a}{3 b}+\frac{b}{6 a} \\
\frac{b}{3 a}-\frac{5 a}{6 b} & \frac{7 a}{3 b}+\frac{b}{3 a} & \frac{b}{6 a}-\frac{7 a}{3 b} & \frac{b}{6 a}-\frac{5 a}{6 b} \\
\frac{5 a}{6 b}-\frac{b}{6 a} & \frac{b}{6 a}-\frac{7 a}{3 b} & \frac{7 a}{3 b}+\frac{b}{3 a} & \frac{5 a}{6 b}-\frac{b}{3 a} \\
\frac{a}{3 b}+\frac{b}{6 a} & \frac{b}{6 a}-\frac{5 a}{6 b} & \frac{5 a}{6 b}-\frac{b}{3 a} & \frac{a}{3 b}+\frac{b}{3 a}
\end{array}\right]} \\
{\left[\begin{array}{cccc}
\frac{a}{3 b}+\frac{b}{3 a} & \frac{a}{6 b}+\frac{b}{3 a} & \frac{-a}{6 b}+\frac{5 b}{6 a} & \frac{a}{3 b}-\frac{5 b}{6 a} \\
\frac{a}{6 b}+\frac{b}{3 a} & \frac{a}{3 b}+\frac{b}{3 a} & \frac{-5 b}{6 a}-\frac{a}{3 b} & \frac{5 b}{6 a}-\frac{a}{6 b} \\
\frac{-a}{6 b}+\frac{5 b}{6 a} & \frac{-5 b}{6 a}-\frac{a}{3 b} & \frac{a}{3 b}+\frac{7 b}{3 a} & \frac{a}{6 b}-\frac{7 b}{3 a} \\
\frac{a}{3 b}-\frac{5 b}{6 a} & \frac{5 b}{6 a}-\frac{a}{6 b} & \frac{a}{6 b}-\frac{7 b}{3 a} & \frac{a}{3 b}+\frac{7 b}{3 a}
\end{array}\right]+\left[\begin{array}{cccc}
\frac{a}{3 b}+\frac{b}{3 a} & \frac{-5 a}{6 b}+\frac{b}{3 a} & \frac{5 a}{6 b}+\frac{5 b}{6 a} & \frac{a}{3 b}-\frac{5 b}{6 a} \\
\frac{-5 a}{6 b}+\frac{b}{3 a} & \frac{7 a}{3 b}+\frac{b}{3 a} & \frac{-5 b}{6 a}-\frac{7 a}{3 b} & \frac{5 b}{6 a}-\frac{5 a}{6 b} \\
\frac{5 a}{6 b}+\frac{5 b}{6 a} & \frac{-5 b}{6 a}-\frac{7 a}{3 b} & 7 \frac{a}{3 b}+\frac{7 b}{3 a} & \frac{-5 a}{6 b}-\frac{7 b}{3 a} \\
\frac{a}{3 b}-\frac{5 b}{6 a} & \frac{5 b}{6 a}-\frac{5 a}{6 b} & \frac{-5 a}{6 b}-\frac{7 b}{3 a} & \frac{a}{3 b}+\frac{7 b}{3 a}
\end{array}\right]}
\end{array}\right]
$$

Typical riser height is twice riser diameter $(H=2 D)$ for top risers opened to atmospheric pressure [14]. The height $(z)$ to diameter $(d)$ ratio used in this work is $2: 1$. Therefore, $b=z=2 \mathrm{~mm}$ and $a=r=0.5 \mathrm{~mm}$ and equation (31) became

$$
\left[K^{e}\right]=\left[\begin{array}{l}
{\left[\begin{array}{cccc}
1.4167 & 1.375 & -0.70833 & 0.75 \\
1.375 & 1.4167 & 0.5833 & 0.625 \\
-0.70833 & 0.5833 & 1.4167 & -1.2917 \\
0.75 & 0.625 & -1.2917 & 1.4167
\end{array}\right]+\left[\begin{array}{cccc}
1.4167 & 1.125 & -0.4583 & 0.75 \\
1.125 & 1.9167 & 0.0833 & 0.4583 \\
-0.4583 & 0.0833 & 1.9167 & -1.125 \\
0.75 & 0.4583 & -1.125 & 1.4167
\end{array}\right]} \\
{\left[\begin{array}{ccccc}
1.4167 & 1.375 & 3.2917 & -3.25 \\
1.375 & 1.4167 & -3.4167 & 3.2917 \\
3.2917 & -3.4167 & 9.4167 & -9.2917 \\
-3.25 & 3.2917 & -9.2917 & 9.4167
\end{array}\right]+\left[\begin{array}{cccc}
1.4167 & 1.125 & -3.5417 & -3.25 \\
1.125 & 1.9167 & -3.9167 & 3.5417 \\
3.5417 & -3.9167 & 1.9167 & -9.5417 \\
-3.25 & 3.5417 & -9.5417 & 1.4167
\end{array}\right]}
\end{array}\right]
$$

Next we evaluated the $\left|f^{e}\right|$ matrix using equation (13)

$$
\left|f^{e}\right|=\int_{T_{1}}^{T_{2}} N\left(U_{1}\right) d \Omega=U_{1} \int_{T_{1}}^{T_{2}} N_{i} d \Omega
$$

The velocity of the molten metal surface entering the riser (boundary $a b$ ) is $U_{1}=1588.6288 \mathrm{~mm} / \mathrm{s}$ [3] and hence the vector $\left|f^{e}\right|$ will be nonzero only for elements 1 and 3 (Figure $1 \mathrm{~b}$ ). These nonzero vectors can be computed as follows [12]:

$$
\left|f^{e}\right|=\int_{T_{1}}^{T_{2}} N\left(U_{1}\right) d \Omega=U_{1} \int_{T_{1}}^{T_{2}} N_{i} d \Omega=\frac{U_{1} \Omega_{i j}}{2}\left[N_{i}\right]
$$

Where $\Omega_{j i}$ denote the lengths of the edge $z$

$$
\left|f^{e}\right|=\left(\frac{U_{1} \Omega_{i j}}{2}\right)\left\{\begin{array}{l}
N_{1}^{1} \\
N_{2}^{1} \\
N_{3}^{1} \\
N_{4}^{1}
\end{array}\right\}=\left(\frac{U_{1} \Omega_{i j}}{2}\right)\left\{\begin{array}{l}
1 \\
0 \\
0 \\
1
\end{array}\right\} ;\left|f^{2}\right|=\left|f^{4}\right|=0 ;\left|f^{3}\right|=\left(\frac{U_{1} \Omega_{i j}}{2}\right)\left\{\begin{array}{l}
N_{1}^{3} \\
N_{2}^{3} \\
N_{3}^{3} \\
N_{4}^{3}
\end{array}\right\}=\left(\frac{U_{1} \Omega_{i j}}{2}\right)\left\{\begin{array}{l}
1 \\
0 \\
0 \\
1
\end{array}\right\}
$$

\subsection{Assembly of the System Matrix}

We assembled the system matrix using figure $1 \mathrm{~b}$ and equation (10)

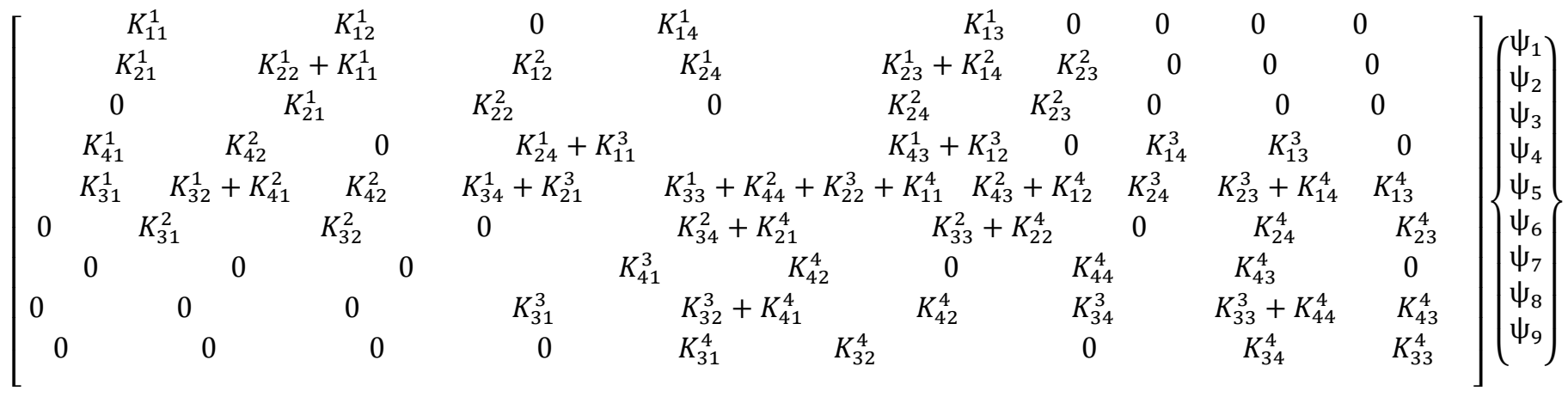


Finally we evaluated the system matrix

$$
=\left(\frac{U_{1} \Omega_{i j}}{2}\right)\left\{\begin{array}{c}
N_{1}^{1} \\
N_{2}^{1}+N_{1}^{2} \\
N_{2}^{2} \\
N_{4}^{1}+N_{1}^{3} \\
N_{3}^{1}+N_{4}^{2}+N_{2}^{3}+N_{1}^{4} \\
N_{3}^{2}+N_{2}^{4} \\
N_{4}^{3} \\
N_{3}^{3}+N_{4}^{4} \\
N_{3}^{4}
\end{array}\right\}
$$

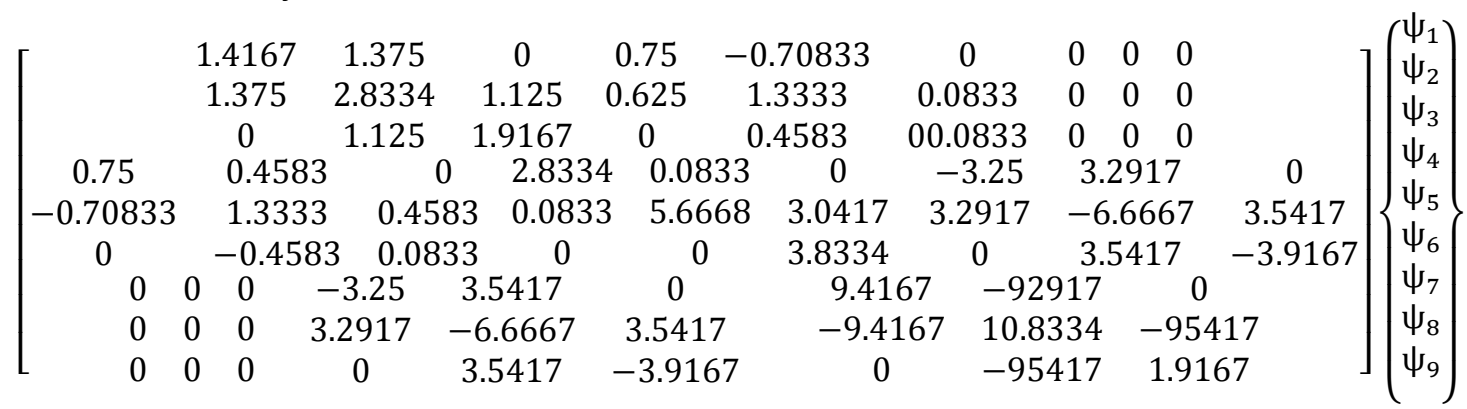

$$
\begin{aligned}
& =\left\{\begin{array}{c}
15888.6288 \\
0 \\
0 \\
3177.2576 \\
0 \\
0 \\
1588.6288 \\
0 \\
0
\end{array}\right\} \\
& \left\{\begin{array}{l}
\psi_{1} \\
\psi_{2} \\
\psi_{3} \\
\psi_{4} \\
\psi_{5} \\
\psi_{6} \\
\psi_{7} \\
\psi_{8} \\
\psi_{9}
\end{array}\right\}=\left\{\begin{array}{c}
568.3382 \\
-898.4816 \\
608.6242 \\
2403.6911 \\
-305.0951 \\
-191.0951 \\
-1054.4221 \\
-59.4082 \\
-122.8500
\end{array}\right\}
\end{aligned}
$$

\section{RESULTS AND DISCUSSION}

Applying the boundary conditions with respect to figure 2 we obtained the following results as shown in Table 1 :

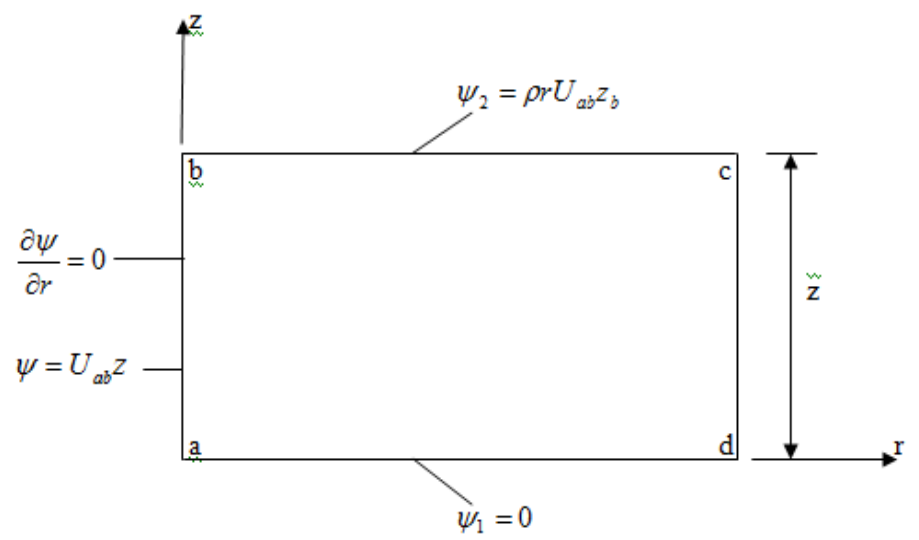

Figure 2: Computational domain and boundary conditions for the stream-function formulation. 
Table 1: Finite Element Results from the Analysis of Axisymmetric Flow of Air through the top riser of Casting using the Stream Function Model

\begin{tabular}{|c|c|c|c|c|c|c|c|}
\hline Nodes & $\begin{array}{l}\mathrm{r} \\
(\mathrm{mm})\end{array}$ & $\begin{array}{l}\mathrm{z} \\
(\mathrm{mm})\end{array}$ & $\begin{array}{l}\text { Stream function } \\
\psi_{F E A}\end{array}$ & $\begin{array}{l}\text { Stream function } \\
\psi_{E X A C T}\end{array}$ & $\begin{array}{l}\text { Velocity }(\mathrm{mm} / \mathrm{s}) \\
u_{F E A}\end{array}$ & $\begin{array}{l}\text { Velocity } \\
\text { (km/hr) }\end{array}$ & $\begin{array}{l}\text { Velocity } \quad(\mathrm{mm} / \mathrm{s}) \\
u_{E X A C T}\end{array}$ \\
\hline 9 & 0 & 0 & 568.3382 & 582.2872 & 0 & 0 & 0 \\
\hline 8 & 0 & 1000 & -898.4816 & - 920.2290 & 0 & 0 & 0 \\
\hline 7 & 0 & 2000 & 608.6242 & 622.6530 & 0 & 0 & 0 \\
\hline 6 & 250 & 0 & 2403.6911 & 2448.0007 & 2775.9873 & 9.9936 & 2823.4303 \\
\hline 5 & 250 & 1000 & -305.0951 & -310.6024 & 8378.6391 & 30.1631 & 8533.9811 \\
\hline 3 & 500 & 0 & 1054.4221 & 1080.3505 & 963.4160 & 3.4683 & 986.1959 \\
\hline 2 & 500 & 1000 & -59.4082 & -59.3844 & 1722.8620 & 6.2023 & 1579.2206 \\
\hline 1 & 500 & 2000 & -122.8500 & -122.6702 & 98.1312 & 0.3533 & 97.8900 \\
\hline
\end{tabular}

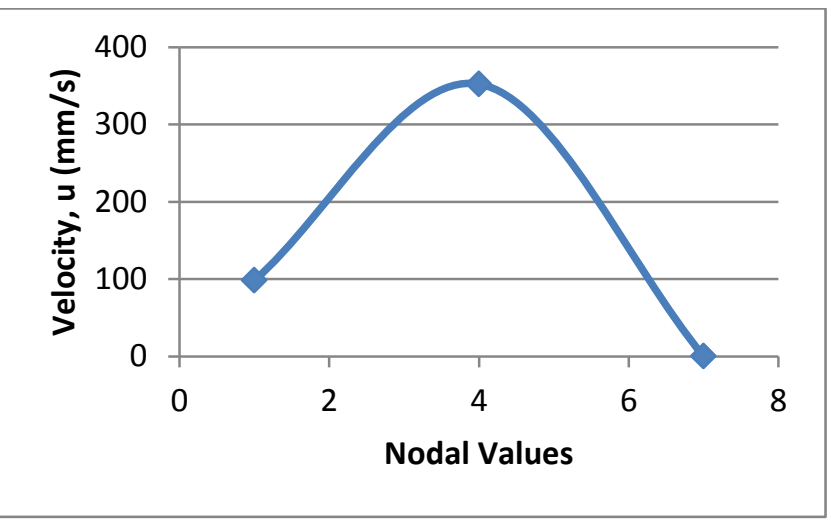

Figure 3(a)

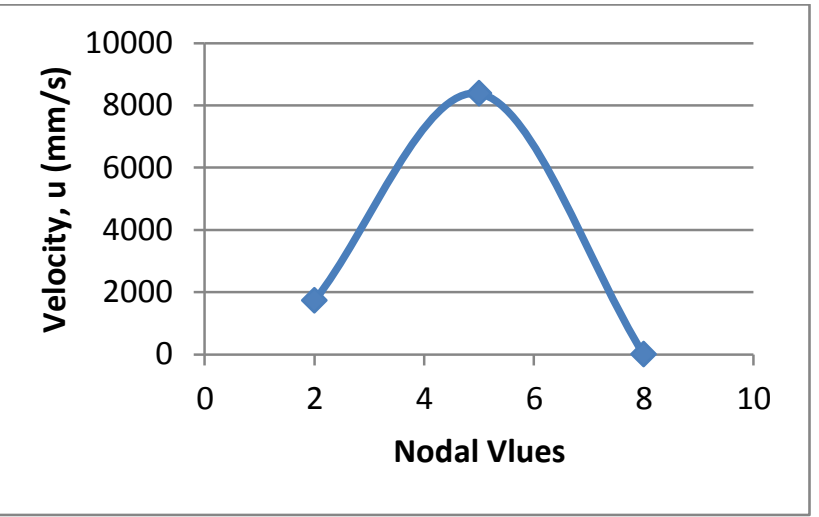

Figure 3(b)

Figure 3: Graph of velocity against nodal values showing (a) the Velocity profile at a cross section along the riser (b) the Velocity profile at a different cross section along the riser

Figures 3 of this work show that the velocity profile at any cross section is parabolic in shape with the maximum velocity at the centre. Figure 4 compares the finite element solution of velocity distribution along the top riser of casting mould at different locations and the exact solution. The result from the finite element analysis converges towards the exact solution.

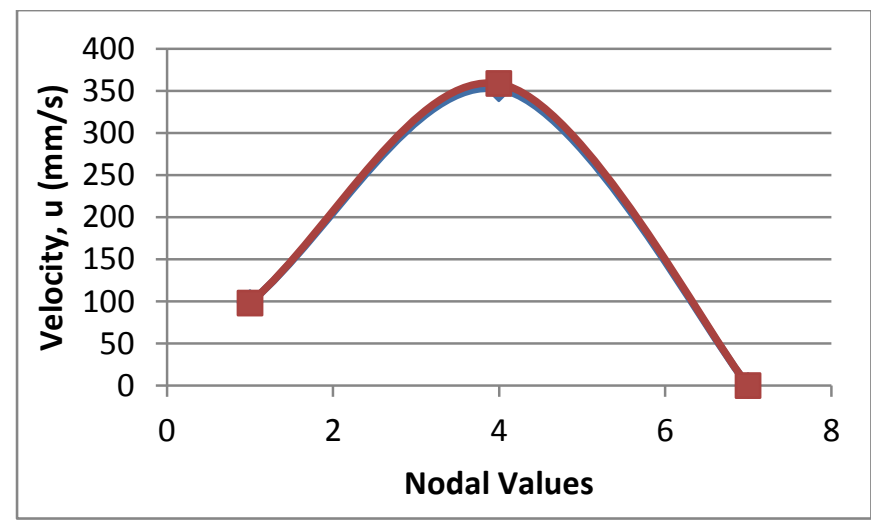

Figure 4: graph of velocity against nodal values showing the Velocity profile at different cross section of the riser this work and Exact solutions

\section{CONCLUSION}

In this work we have used the finite element method and the stream function model to analyze the axisymmetric flow of air through the top risers of casting. Results show that the velocity profile at any cross section is parabolic in shape with the maximum velocity at the centre. Comparing results with exact solution shows that the finite element result converged towards the exact solution. Before now all researches do was to develop empirical equations and optimized molten metal flow in casting mould. This work has gone further to analyze the behaviour of air flow in casting mould.

\section{REFERENCES}

[1] Ravi B. Metal Casting: Computer-Aided Design and Analysis, Prentice-Hall, New Delhi, India, 2005.

[2] Feng, L. Optimized Design of Gating/Riser System in Casting Based on CAD and Simulation Technology. MSc. Thesis, Worchester Polytechnic Institute, 2008.

[3] Inegbedion F. and Akpobi J.A.: "Determination of the Compressibility of Air in Casting Mould", Nigerian Research Journal of Engineering and 
Environmental Sciences, vol. 2, No. 2,pp. $524-$ 529, 2017.

[4] Bradley F. J. and Heinmann F. J: "A Hydraulics Based Model of Fluid Flow in Horizontal Gating System", AFS Transactions, Vol.101, pp.917-923, 1992.

[5] Campbell J. Materials Solutions Conference on Aluminium Casting Technology, Chicago, 1998.

[6] Campbell J.Castings, Elsevier ButterworthHeinemann, 2003.

[7] Runyoro J, Boutorabi S. M. A., Campbell J. “Critical Gate Velocity for Film Forming Casting Alloys, a Basis for Process Specification", AFS Transactions, vol. 100, pp 225-234, 1992.

[8] Svoboda J. M. American Foundry Mens Society Cast Metals Institute AFS-CMI., 1995.

[9] Yang X., Jolly M. and Campbell J.MCWASP IX, 420427, 2000.
[10] Reddy J. N. An Introduction to the Finite Element Method, Second Edition, McGraw-Hill Inc., 1993.

[11] Reddy J. N. An Introduction to the Finite Element Method, Third Edition, International Edition McGraw-Hill Inc., 2006.

[12] Singiresu S. Rao. The Finite Element Methods in Engineering, Fourth Edition, Elsevier Science and Technology Books Publisher, 2004.

[13] Inegbedion F. and Akpobi J. A. "Stream Function Modelling of Compressible Axisymmetric Flow of Air through the Riser of Casting Mould"; Transactions of the Nigerian Association of Mathematical Physics, volume 5, 2017, pp. 149 152.

[14] Flinn, R. A. Fundamentals of Metal Casting, A'ddison-Wesley Publishing Co., 1963. 